\title{
On the Analysis of Non-stationary Impedance Spectra
}

\author{
Alberto Battistel, ${ }^{*[a]}$ Guoqing Du, ${ }^{[\mathrm{b}]}$ and Fabio La Mantia*[b]
}

\begin{abstract}
In this work we study the possibility to analyze the non-stationary impedance spectra by employing standard equivalent circuits. For this purpose, the dynamic multi-frequency analysis (DMFA) is introduced and compared with a set of consecutive stationary impedance spectra. In order to prove the hypothesis, the data are obtained by the simulation in the time domain of the electron transfer process between an electrode and a free-diffusing redox couple in solution. During the simulation, the system is perturbed with a cyclic voltammetry super-
\end{abstract}

imposed to a small multisine perturbation (DMFA) or with a series of stationary impedance spectroscopies. Also, a new fitting algorithm, which takes into account the correlation between consecutive spectra, is proposed and tested. Although the Randles circuit can be used to fit both dynamic and stationary impedance spectra, the values of the fitting parameters are different and depend on the direction of the scan and on the rate. This is related to the influence of the diffusion profile on the fitting parameters.

Keywords: multisine design $\cdot$ dynamic electrochemical impedance spectroscopy $\cdot$ electrochemical impedance spectroscopy $\cdot$ Fourier analysis $\cdot$ least squares fitting algorithm.

\section{Introduction}

Does it make sense to make impedance spectroscopy in non-stationary conditions? And if yes, how should one analyze the results? The main goal of this work is to give a satisfactory answer to these two questions.

Regarding the first question and according to the usual interpretation of the concept of impedance, the answer should be no, because impedance is not defined as timedependent and, therefore, there should not exist an impedance out of stationary conditions. However, under some conditions, it is possible to show that time dependence can be conciliated into the concept of impedance. The mathematical formulation of this problem is presented in this article.

In literature it is possible to find some works which deal with the concept of dynamic impedance.

Ragoisha et al. introduced the idea of potentiodynamic electrochemical impedance spectroscopy (PDEIS) [1]. This technique is based on a subsequent set of impedance spectra measured on stationary conditions on a staircase potential profile. The impedance is collected in the standard fashion, a single frequency at the time. The literature is full of examples of series of impedance spectra used to study an electrochemical reaction in a range of potential. Some noticeable examples are the Fourier transform ac voltammetry employed especially by Bond and coworkers (for example reference [2-4]) and the Fourier transforms electrochemical impedance spectroscopy (FT-EIS) reported also by Pettit and Roy (some examples $[5,6])$. In both cases, a multisine signal is superimposed to a step by step potential ramp and the impedance is calculated through Fourier analysis.

Interpretation of the results in these cases is achieved though fitting of the spectra with an equivalent circuit which describes the physico-chemical behavior of the system or simply provides a rational series of parameters which are then studied as function of the potential.

Darowicki achieved an instantaneous impedance spectrum using short-time Fourier transforms (STFT) [7-11]. In this technique a multisine is superimposed to a true linear potential ramp and the data are recorded in continuous. The impedance is then calculated breaking the recorded data in blocks and performing STFT on every block. The STFT have the advantage that can capture both the frequency and the time dependence of the signals. In this way it is possible to recover sets of impedance spectra in a range of frequencies as function of time.

Sacci and Harrington used very similar approach in what they called dynamic electrochemical impedance spectroscopy (dEIS) [12-15]. They did not investigate the properties of the STFT, but provided valuable discussions regarding the problem of performing Fourier analysis on a dynamic system. They implemented a baseline correction to compensate for the evolution of the system and discussed which frequency constrain should be taken in superimposing a frequency signal on a linear voltammetry [15].

\section{[a] A. Battistel}

Laboratoire d'Electrochimie Physique et Analytique (LEPA) École Polytechnique Fédérale de Lausanne (EPFL),

Switzerland

*e-mail: alberto.battistel@epfl.ch

[b] G. Du, F. La Mantia

Energiespeicher- und Energiewandlersysteme

Universität Bremen, 28359 Bremen, Germany *e-mail:lamantia@uni-bremen.de

Supporting information for this article is available on the WWW under http://dx.doi.org/10.1002/elan.201600260. 
Harrington discussed also the implication of KramerKronig (KK) relationships in the case of dynamic impedance [14]. He pointed out that the stability, implied in the KK relationships, is connected with the absence of negative time constants in a system. This guarantees that, upon small perturbation, the system will not diverge away from its steady state condition.

Hubin et al. developed the odd random phase EIS (ORP-EIS) [16-20]. They employ a multisine composed by odd harmonics of a base frequency, removing every third frequency. This expedient allows quantifying the non-linear behavior of the system, as well as the instability [19]. Using the ORP-EIS, they were able to correct the effect of the time-evolution of the system on the impedance spectra. By acquisition of consecutive data sets, they were able to reconstruct the variation of the impedance with time. However, they needed to assume a particular shape of the time variation of the impedance. The final results were modeled using equivalent circuits.

It still remains the question on how to analyze the large amount of impedance spectra collected during dynamic impedance. Of course the most naïve approach would be to perform a fitting per spectrum, but in this way the correlation among the values of the fitting parameters describing consecutive spectra would be lost.

In this work we show and discuss the possibility to use standard equivalent circuits for fitting the impedance spectra, as well as an advanced algorithm for handling the time variation of the fitting parameters. The algorithm handles all the spectra at the same time and accounts also for their correlation, taking into account that the parameters have to change smoothly in the time domain.

This algorithm was already employed with good results [21]; in this work it will be explained in more details. In order to prove the validity of this approach, simulated data were used. The simulations are based on a simple physical model of a redox couple in solution, which is perturbed by a triangular voltage profile (cyclic voltammetry), superimposed to a multi-sine signal. The data were simulated in the time domain. By using simulated data, it is possible to attribute the results of the fitting procedure and data extraction fully to the technique, and not to experimental limitations.

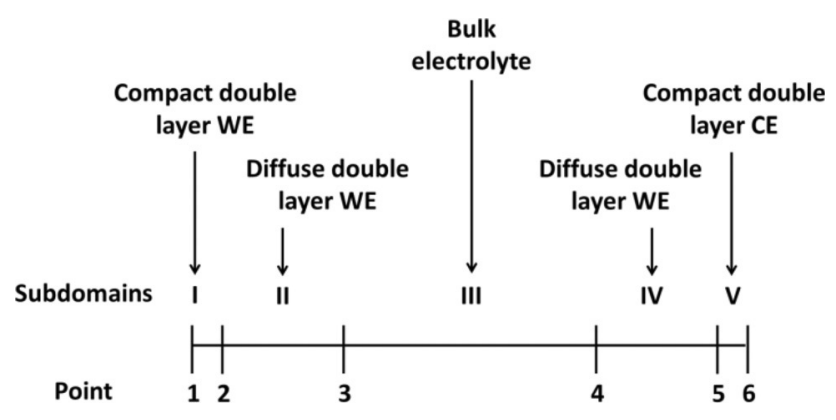

Fig. 1. Schematic drawing of the simulation domain.

\section{Experimental}

\subsection{Details of the Simulation}

Simulations were performed using COMSOL Multiphysics 4.4 based on the finite element method. Current and voltage were calculated in the time domain. The 1-D model is divided in 5 subdomains, which describe the compact double layer of the two electrodes, the diffuse double layer/electron transfer region of the two electrodes, and the bulk solution (see Figure 1). In Table 1 the differential equations describing the subdomains and the boundary conditions are reported. The compact double layers do not contain charged species and the dielectric constant, $\varepsilon_{\mathrm{r}}$, in these subdomains is equal to 10 . The electron transfer is simulated by an electron tunneling, which can reach a certain distance from the outer Helmholtz plane (OHP).

The electron transfer rate decreases exponentially with the distance from the OHP and is located in subdomains II and IV. The two electrodes are the same, and have therefore the same Fermi level. The Fermi level of the solution is located $0.05 \mathrm{eV}$ above the Fermi level of the metal. The dielectric constant of the medium in subdomains II-IV is equal to 72 . The values of the physical constants used in the simulations are reported in Table 2. The concentration of reduced and oxidized species was set equal to $10 \mathrm{mM}$, and the value of diffusion coefficients and oxidation number for $\left[\mathrm{Fe}(\mathrm{CN})_{6}\right]^{4-}$ and $\left[\mathrm{Fe}(\mathrm{CN})_{6}\right]^{3-}$ were used, while the concentration of the supporting elec-

Table 1. List of the differential equations describing the subdomains and their boundary conditions.

\begin{tabular}{lll}
\hline Phenomenon & Equation & Subdomains \\
\hline Mass balance & $\frac{\partial \mathrm{c}_{\mathrm{i}}}{\partial \mathrm{t}}=\mathrm{D}_{\mathrm{i}} \cdot \nabla^{2}\left(\mathrm{c}_{\mathrm{i}}\right)+\frac{\mathrm{z}_{\mathrm{i}} \mathrm{FD}}{\mathrm{RT}} \cdot \nabla\left(\mathrm{c}_{\mathrm{i}} \nabla \varphi\right)+\mathrm{G}_{\mathrm{i}}$ & II-IV \\
Charge balance & $\varepsilon_{0} \varepsilon_{\mathrm{r}} \nabla^{2}(\varphi)=\mathrm{F} \cdot \sum_{\mathrm{i}} \mathrm{z}_{\mathrm{i}} \mathrm{c}_{\mathrm{i}}$ & I-V \\
Charge transfer & $\mathrm{G}_{\mathrm{red}}(\mathrm{x})=\mathrm{k}^{0} \exp \left[-\beta\left|\mathrm{x}-\mathrm{x}_{\mathrm{OHP}}\right|\right]\left\{\mathrm{c}_{\mathrm{ox}} \exp \left[-\frac{\alpha \mathrm{F} \eta(\mathrm{x})}{\mathrm{RT}}\right]-\mathrm{c}_{\mathrm{red}} \exp \left[\frac{(1-\alpha) \mathrm{F \eta}(\mathrm{x})}{\mathrm{RT}}\right]\right\}$ & $\mathrm{II}$ and IV \\
\hline Phenomenon & Equation & Points \\
\hline Blocking surface & $-\left[\mathrm{D}_{\mathrm{i}} \cdot \nabla\left(\mathrm{c}_{\mathrm{i}}\right)+\frac{\mathrm{z}_{\mathrm{i}} \mathrm{FD}_{\mathrm{i}}}{\mathrm{RT}} \cdot \mathrm{c}_{\mathrm{i}} \nabla \varphi\right]=0$ & 2 and 5 \\
Continuity & & 3 and 4 \\
Grounded WE & $\varphi=0$ & 1 \\
Floating CE & $\varphi=-\Delta \mathrm{E}-\Delta \mathrm{E}_{\mathrm{F}}$ & 6 \\
\hline
\end{tabular}


Table 2. Physical constants used in the simulations.

\begin{tabular}{lll}
\hline Name & Meaning & Value \\
\hline$\alpha$ & Symmetry factor & 0.5 \\
$\beta$ & Inverse of the tunnelling distance & $5 \cdot 10^{9}\left[\mathrm{~m}^{-1}\right]$ \\
$\mathrm{d}_{\mathrm{h}}$ & Length of Sub-domain I & $5 \cdot 10^{-10}[\mathrm{~m}]$ \\
$\mathrm{D}_{\mathrm{F}}$ & Diffusion coefficient of $\mathrm{F}^{-}$ & $1.48 \cdot 10^{-9}\left[\mathrm{~m}^{2} \mathrm{~s}^{-1}\right]$ \\
$\mathrm{D}_{\mathrm{K}}$ & Diffusion coefficient of $\mathrm{K}^{+}$ & $1.96 \cdot 10^{-9}\left[\mathrm{~m}^{2} \mathrm{~s}^{-1}\right]$ \\
$\mathrm{D}_{\text {ox }}$ & Diffusion coefficient of Ox & $8.96 \cdot 10^{-10}\left[\mathrm{~m}^{2} \mathrm{~s}^{-1}\right]$ \\
$\mathrm{D}_{\text {red }}$ & Diffusion coefficient of Red & $7.35 \cdot 10^{-10}\left[\mathrm{~m}^{2} \mathrm{~s}^{-1}\right]$ \\
$\mathrm{k}^{0}$ & Rate constants & $10^{6}\left[\mathrm{~s}^{-1}\right]$ \\
$\mathrm{L}$ & Length of simulation domain & $1 \cdot 10^{-4}[\mathrm{~m}]$ \\
$\mathrm{T}$ & Temperature & $298.15[\mathrm{~K}]$ \\
\hline
\end{tabular}

trolyte was set equal to $500 \mathrm{mM}$, and the value of diffusion coefficients and oxidation number for KF were used.

\subsection{How to Construct a Multi-frequency Signal}

One of the requirements to recover an impedance spectrum in dynamic conditions is that all the parts of the spectrum are subjected to the same dynamic condition. Therefore, all the frequencies at which the impedance is recorded should be injected simultaneously into the system through a multisine. The multisine should guarantee the same advantages in terms of accuracy and resolution as a standard EIS performed with a subsequent set of frequencies.

The distribution of frequencies of the multisine should be as close as possible to a true logarithmic distribution, as in classic impedance measurements. However, it is necessary that the single frequencies of the multisine are harmonics of a common base frequency, $\mathrm{f}_{\mathrm{b}}$. This frequency should be at least an order of magnitude smaller than all the others, in order to guarantee a satisfying shape of the signal.

In designing a multisine one should also look to the interaction among different frequencies, i.e. higher harmonics and intermodulation bands. Since the system is nonlinear, it is expected that at least the fundamental and second harmonics for each frequency will appear. Furthermore, any frequency could intermodulate with each other, as it is evident from Figure 2 (see the peak at $4 \mathrm{~Hz}$ ). It is therefore fundamental to seek a good distribution of frequencies.

Starting from a quasi-logarithmic distribution, in which all frequencies are multiple of $f_{b}$, we expressed the constrain of non-interfering as:

$\mathrm{f}_{\mathrm{i}} \pm \mathrm{f}_{\mathrm{j}} \pm \mathrm{f}_{\mathrm{k}} \neq \mathrm{f}_{\mathrm{m}} \quad$ with $\mathrm{m} \neq \mathrm{i}, \mathrm{j}, \mathrm{k}$

where $\mathrm{i}, \mathrm{j}, \mathrm{k}$, and $\mathrm{m}$ are the indexes of the frequencies composing the multi-frequency signal. In the case this constrain cannot be respected, the frequency $f_{m}$ is removed from the multisine. In this work the multisine covered 4 decades with 35 frequencies.

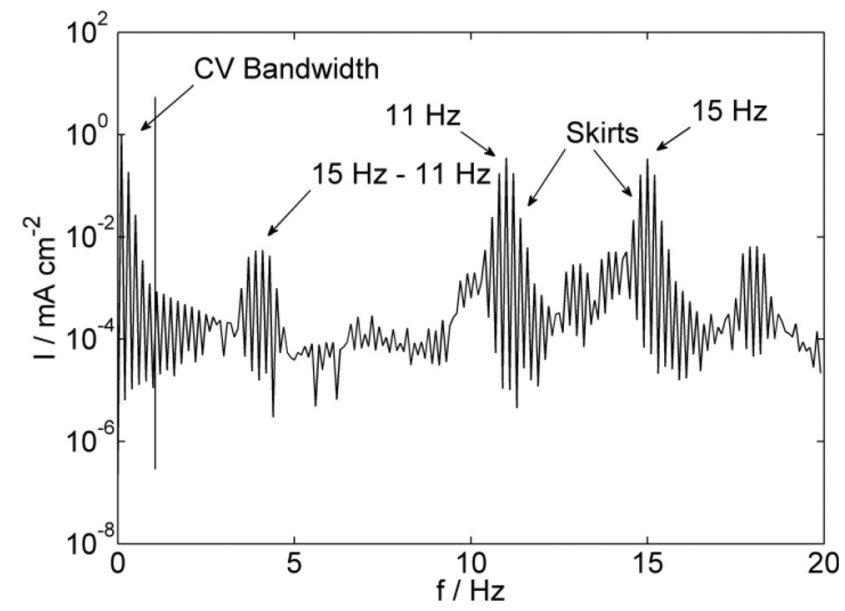

Fig. 2. Low frequency region of the frequency domain of the current during multisine cyclic voltammetry $\left(100 \mathrm{mV} \mathrm{s}^{-1}\right.$ between $-0.25 \mathrm{~V}$ and $0.25 \mathrm{~V}$ ) showing the extension of the $\mathrm{CV}$ bandwidth in the current and the first two signals of the multisine (11 and $15 \mathrm{~Hz}$ ) with some intermodulation peaks $(4 \mathrm{~Hz})$.

It is common use in the design of multisine to modify the amplitude of the single perturbations in order to reduce the crest factor. The usual way is to decrease the amplitude of the highest frequencies, because they have the lowest impedance value. The multisine used here employs a square root law for the amplitudes $A_{k}$ :

$\mathrm{A}_{\mathrm{k}}=\mathrm{A}_{0} \cdot\left(1+\frac{70}{\sqrt{\mathrm{f}_{\mathrm{k}}}}\right)$

The last parameter to optimize is the phase of the single frequencies in such a way to decrease the crest factor $\mathrm{Cr}$ which we defined as:

$$
\mathrm{Cr}=\frac{\max \left[\operatorname{abs}\left(\mathrm{v}_{\mathrm{ac}}\right)\right]}{\sqrt{\frac{1}{\mathrm{~T}} \int_{0}^{\mathrm{T}} \mathrm{v}_{\mathrm{ac}}^{2}(\mathrm{t}) \mathrm{dt}}}
$$

where $\mathrm{v}_{\mathrm{ac}}$ is the multisine perturbation and $\mathrm{T}$ is the total time. The lower the crest factor, the lower the power injected into the system, which guarantees a behavior of the system closer to linearity. The multisine used in this work had peak-to-peak amplitude, after phase optimization, of $50 \mathrm{mV}$, and a crest factor equal to 2.4 .

\subsection{Theory of Multisine Cyclic Voltammetry}

During dynamic multi-frequency analysis the impedance spectra are measured in dynamic conditions, i.e. when the system is non-stationary. It is important to distinguish between the time scale of the cyclic voltammetry and that of the impedance spectrum. The first encloses all the phenomena controlled by the actual scan rate of the voltammetry. The latter represents the time scale at which a single impedance point is effectively recorded. It is im- 
portant that these time scales do not overlap. In the frequency domain, the time scale is translated into the concept of bandwidth of a signal or event, which represents how broad is the range of frequencies interested by the signal. Large time scales correspond to small bandwidth and vice versa.

As example, a CV can range between tenth to hundreds of seconds which correspond to a bandwidth from $10 \mathrm{~Hz}$ to few MHz. In this case the time scale of the impedance spectra should be limited to less than a tenth of second, which means that the lower frequency should be larger than $10 \mathrm{~Hz}$. In this way the time scale of the CV will not overlap with the time scale of the multisine perturbation (see Figure 2). Under this assumption it is possible to recover one integral impedance spectrum at each point in time. The actual voltage perturbation injected into the system is:

$\mathrm{v}(\mathrm{t})=\mathrm{v}_{\mathrm{CV}}(\mathrm{t})+\sum_{\mathrm{k}=1}^{\mathrm{N}_{\mathrm{f}}} \mathrm{v}_{\mathrm{k}}(\mathrm{t})$

which is the sum of the cyclic voltammetry perturbation:

$\mathrm{v}_{\mathrm{CV}}(\mathrm{t})=\mathrm{v}_{0}+\sum_{\mathrm{l}=1}^{\mathrm{N} / 2} \operatorname{Re}\left[\mathrm{V}_{\mathrm{CV}, \mathrm{l}} \cdot \exp (2 \pi \cdot \mathrm{j} \cdot \mathrm{l} \cdot \Delta \mathrm{f} \cdot \mathrm{t})\right]$

where $\mathrm{v}_{0}$ is the middle point of the voltammetry, $\mathrm{N}$ is the total number of samples recorded, $\mathrm{V}_{\mathrm{CV}, 1}$ is the l-th component of the Fourier transform of the cyclic voltammetry, and $\Delta \mathrm{f}$ is the frequency resolution given by the inverse of T. To avoid spectrum leakage an integer number of voltammograms and multisine oscillations should be taken. By using a filter, the summation in eq. (5) is truncated at a certain term.

The second term in eq. 4 is the multisine perturbation, given by the sum of all the single perturbations at frequency $f_{k}$ at which the impedance is measured:

$\mathrm{v}_{\mathrm{k}}(\mathrm{t})=\operatorname{Re}\left[\mathrm{V}_{\mathrm{k}} \cdot \exp \left(2 \cdot \pi \cdot \mathrm{j} \cdot \mathrm{f}_{\mathrm{k}} \cdot \mathrm{t}\right)\right]$

where $\mathrm{V}_{\mathrm{k}}$ is the Fourier transform of the voltage at frequency $\mathrm{f}_{\mathrm{k}}$. As the voltage, also the current can be divided in terms related to the cyclic voltammetry and to the multisine perturbation:

$\mathrm{i}(\mathrm{t})=\mathrm{i}_{\mathrm{CV}}(\mathrm{t})+\sum_{\mathrm{k}=1}^{\mathrm{N}_{\mathrm{f}}} \mathrm{i}_{\mathrm{k}}(\mathrm{t})$

where the component corresponding to the cyclic voltammetry can be expressed as:

$\mathrm{i}_{\mathrm{CV}}(\mathrm{t})=\mathrm{i}_{0}+\sum_{\mathrm{l}=1}^{\mathrm{N} / 2} \operatorname{Re}\left[\mathrm{I}_{\mathrm{CV}, 1} \cdot \exp (2 \cdot \pi \cdot \mathrm{j} \cdot \mathrm{l} \cdot \Delta \mathrm{f} \cdot \mathrm{t})\right]$ $\mathrm{i}_{0}$ represents the average current and the summation is the expansion in Fourier series of the current flowing during the cyclic voltammetry. The current generated by the multisine perturbation at frequency $f_{k}$ is given by the sum of the linear component, which represents the average current at $\mathrm{f}_{\mathrm{k}}$, and the intermodulation of the frequency $\mathrm{f}_{\mathrm{k}}$ with the cyclic voltammetry:

$$
\begin{aligned}
& \mathrm{i}_{\mathrm{k}}(\mathrm{t})=\mathrm{i}_{\mathrm{k}, 0}(\mathrm{t})+\sum_{\mathrm{l}=1}^{\mathrm{N} / 2} \operatorname{Re}\left\{\mathrm{I}_{\mathrm{k}, 1} \cdot \exp \left[2 \cdot \pi \cdot \mathrm{j} \cdot\left(\mathrm{f}_{\mathrm{k}}-\mathrm{l} \cdot \Delta \mathrm{f}\right) \cdot \mathrm{t}\right]\right\}+ \\
& +\sum_{\mathrm{l}=1}^{\mathrm{N} / 2} \operatorname{Re}\left\{\mathrm{I}_{\mathrm{k}, 1} \cdot \exp \left[2 \cdot \pi \cdot \mathrm{j} \cdot\left(\mathrm{f}_{\mathrm{k}}+\mathrm{l} \cdot \Delta \mathrm{f}\right) \cdot \mathrm{t}\right]\right\}
\end{aligned}
$$

where $i_{k, 0}$ is the average current of a single frequency perturbation during the cyclic voltammetry:

$\mathrm{i}_{\mathrm{k}, 0}(\mathrm{t})=\operatorname{Re}\left[\mathrm{I}_{\mathrm{k}} \cdot \exp \left(2 \cdot \pi \cdot \mathrm{j} \cdot \mathrm{f}_{\mathrm{k}} \cdot \mathrm{t}\right)\right]$

which is the only term remaining in a classic stationary impedance. The second and third terms in eq. 9 represent the skirt around the frequency $\mathrm{f}_{\mathrm{k}}$ (see Figure 2) and it is given by the non-linear behavior of the system. This represents the intermodulation of the frequency $\mathrm{k}$ with the cyclic voltammetry, a generalization of what we have already proposed [22-24]. As underlined by Breugelmans, the skirt is the primary evidence of time instability [19]. In Figure 2, some skirts are visible around the first two frequencies of the multisine at 11 and $15 \mathrm{~Hz}$.

By using an appropriate filter it is possible to separate for each frequency $\mathrm{f}_{\mathrm{k}}$ the value of $\mathrm{v}_{\mathrm{k}}(\mathrm{t})$ and $\mathrm{i}_{\mathrm{k}}(\mathrm{t})$ and calculate the impedance $Z_{k}$. The filter effectively truncates all the summations to a value bw $\ll \mathrm{N} / 2$. A too large filter would incorporate too many unwanted signals (harmonics, intermodulations, and neighboring perturbations) and one too small would distort the time evolution of the signals. The best choice is to match the bandwidth of the filter with the base frequency of the multisine signal, and take a base frequency at least 10 times higher than the frequency of the cyclic voltammetry. In this way the same filter, with identical bandwidth can be used to recollect also the shape of the cyclic voltammetry for the DMFA experiments (or simulations). The impedance $Z_{k}$ at the frequency k can be written as:

$\mathrm{Z}_{\mathrm{k}}(\mathrm{t})=\frac{\mathrm{V}_{\mathrm{k}}}{\mathrm{I}_{\mathrm{k}}} \cdot\left\{1-\sum_{\mathrm{l}=1}^{\mathrm{bw}}\left[\begin{array}{l}\frac{\mathrm{I}_{\mathrm{k}, 1}}{\mathrm{I}_{\mathrm{k}}} \cdot \exp [2 \cdot \pi \cdot \mathrm{j} \cdot \mathrm{l} \cdot \Delta \mathrm{f} \cdot \mathrm{t}] \\ +\frac{\mathrm{I}_{\mathrm{k}, 1}}{\mathrm{I}_{\mathrm{k}}} \cdot \exp [-2 \cdot \pi \cdot \mathrm{j} \cdot \mathrm{l} \cdot \Delta \mathrm{f} \cdot \mathrm{t}]\end{array}\right]\right\}$

where the first term corresponds to the average impedance during the $\mathrm{CV}$ and the summation represents the time dependency of the system at that frequency. The bandwidth of $Z_{k}$ is defined by the filter. $Z_{k}$ possesses an actual value at any point in time which is an integral 
value given by the bandwidth of the filter. In plain words, if the bandwidth of the filter is $1 \mathrm{~Hz}$ then the value of $\mathrm{Z}_{\mathrm{k}}$ is the integral weighted on one second according to the shape of the filter. In this work we used a box filter. Following eq. 11 , the impedance $Z_{k}$ has a value for every sample recorded (5 million in this work), and an additional integration was used to reduce the number of samples to 200 .

\subsection{Fitting Procedure}

In order to fit the large amount of impedance spectra obtained during the DMFA experiments in a consistent way, the classic algorithm, based on the non-linear least squares minimization, is not sufficient. The main limitation is related to the fact that the fitting parameters do not account for their correlation and can change drastically among the spectra. In order to preserve their correlation and obtain smooth trends, a function $S_{P}$ is minimized simultaneously to the least square. The function $S_{P}$ is defined as follows:

$\mathrm{S}_{\mathrm{P}}=\frac{\left(\begin{array}{l}2 \mathrm{P}_{1}-5 \mathrm{P}_{2}+4 \mathrm{P}_{3}-\mathrm{P}_{4}+\sum_{\mathrm{i}=2}^{\mathrm{N}-1}\left(\mathrm{P}_{\mathrm{i}+1}+\mathrm{P}_{\mathrm{i}-1}-2 \mathrm{P}_{\mathrm{i}}\right)^{2} \\ -\mathrm{P}_{\mathrm{N}-3}+4 \mathrm{P}_{\mathrm{N}-2}-5 \mathrm{P}_{\mathrm{N}-1}+2 \mathrm{P}_{\mathrm{N}}\end{array}\right)}{\sum_{\mathrm{i}=1}^{\mathrm{N}}\left(\mathrm{P}_{\mathrm{i}}\right)^{2}}$

where $\mathrm{P}$ is the parameter, $\mathrm{N}$ is the total number of impedance spectra, and the index i represents the index of the impedance spectra. Eq. (12) represents the numerical integral of the second derivative of the parameter $\mathrm{P}$ with respect to the index of the impedance. By minimizing $S_{P}$ the algorithm minimizes the number of times that the curve is changing concavity, i.e. the number of peaks present in the curve. By defining the classic $\chi_{i}^{2}$ of the i-th impedance spectrum as:

$$
\begin{array}{r}
{\left[\operatorname{Re}\left(Z_{i}\left(\omega_{1}\right)\right)-\operatorname{Re}\left(Z_{i, 1}\right)\right]^{2}} \\
\chi_{i}^{2}=\sum_{l=1}^{N_{f}} \frac{+\left[\operatorname{Im}\left(Z_{i}\left(\omega_{1}\right)\right)-\operatorname{Im}\left(Z_{i, 1}\right)\right]^{2}}{\left[\operatorname{Re}\left(Z_{i, 1}\right)\right]^{2}+\left[\operatorname{Im}\left(Z_{i, 1}\right)\right]^{2}}
\end{array}
$$

we minimized the following function:

$$
\chi_{\mathrm{T}}^{2}=\sum_{\mathrm{i}=1}^{\mathrm{N}} \chi_{\mathrm{i}}^{2}+\sum_{\mathrm{P}=1}^{\mathrm{N}_{\mathrm{P}}} \mathrm{w}_{\mathrm{P}} \cdot \mathrm{S}_{\mathrm{P}}
$$

where $\mathrm{N}_{\mathrm{P}}$ is the total number of parameters. The algorithm is attempting to select the fitting parameters in order to obtain a good fitting of the impedance spectra and simultaneously having smooth variation of the parameters with the potential (or time). Moreover, by selecting the value of $\mathrm{w}_{\mathrm{P}}$ it is possible to decide if a parame- ter should change more smoothly than another one. The software is written in such a way that if $\mathrm{w}_{\mathrm{P}}$ is set equal to infinite, the parameter $\mathrm{P}$ will be considered the same for all impedance spectra.

Although all the spectra are fitted simultaneously and additional constrains are added, the total computational time for the algorithm was below 15 minutes for 41 spectra on a standard computer, which is similar, if not shorter, than performing 41 single fittings.

\section{Results and Discussion}

The simulated system, composed by two electrodes in contact with a solution containing a redox couple, was subjected subsequently to a set of stationary impedance spectra and to a dynamic multi-frequency analysis. The stationary impedances were performed in the most standard fashion, waiting ten seconds at each voltage step for the stabilization of the system and superimposing subsequently several frequencies, starting from the highest and ending with the lowest. In this way every spectrum requires circa $12 \mathrm{~s}$, with a total duration of the experimental equal to 10 minutes. On the other hand, during the DMFA all the frequencies were injected simultaneously into the system by superimposing the multisine signal to the voltage profile of the cyclic voltammetry. The experiment in this way has an estimated total duration of $10 \mathrm{~s}$, given by the period of the cyclic voltammetry. Figure 3 shows some examples of the stationary impedances measured between $-100 \mathrm{mV}$ and $+100 \mathrm{mV}$ with the corresponding fitting. In this work, all the spectra (both dynamic and stationary) were fitted using the Randles circuit:

$\mathrm{R}_{\mathrm{s}}+\mathrm{C}_{\mathrm{h}} /\left(\mathrm{R}_{\mathrm{ct}}+\mathrm{W}\right)$

where $R_{s}$ is the resistance of the electrolyte, which represents the ohmic loss due to the electrolyte, $C_{h}$ is the differential interface capacitance, accounting for the storage of charge at the interface, $\mathrm{R}_{\mathrm{ct}}$ is the charge transfer resistance, which considers the kinetic limitation of the charge

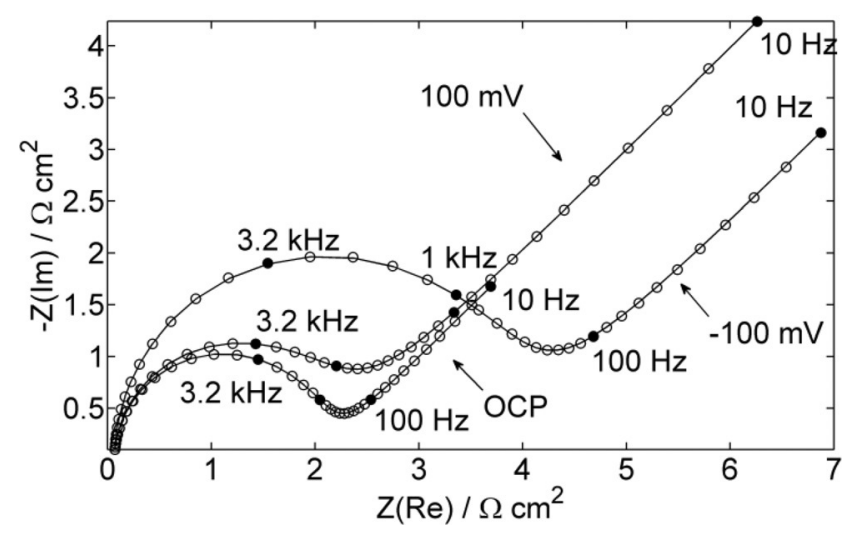

Fig. 3. Nyquist plot for three static impedances with corresponding fitting at $-100 \mathrm{mV}, 0$, and $100 \mathrm{mV}$. 
transfer reaction, and $\mathrm{W}$ is the Warburg element, defined as $\mathrm{W}=\sigma(\mathrm{j} \omega)^{-0.5}$, which represents the effect of the diffusion of species toward the interface. The symbols + and / correspond to serial and parallel connection, respectively. The Randles circuit assumes also that capacitive and faradaic currents do not interact and can be treated separately. The spectra in Figure 3 show the typical semicircle followed by a $45^{\circ}$ straight line as expected by the Randles circuit. The curves are neat and the fitting is very good with a total value of $\chi_{\mathrm{T}}^{2}$ equal to $4.3 \cdot 10^{-5}$. The fitting was performed considering all the spectra at the same time and allowing for some extra smoothing conditions between every element to fit, in such a way that every element has to change smoothly between consecutive spectra.

Some of the spectra of the DMFA are reported in Figure 4 together with their fitting. These spectra are taken during cathodic scan. The points at lower frequencies are more scattered than in the case of the stationary impedance. In fact, as also clear from Figure 2, some intermodulation peaks appear very close to the first multisine frequencies, introducing some distortions. This phenomenon is stronger at lower frequencies where the distance between consecutive frequencies is lower. Although the spectra are more scattered, the fitting performed well, with a value of $\chi_{\mathrm{T}}^{2}$ equal to $4.7 \cdot 10^{-4}$ and $3.4 \cdot 10^{-4}$ for the cathodic and for the anodic scan, respectively. These values are larger than that reported for the fitting of the stationary impedance, because of the quality of the data; however, they are good enough to state that a Randles type equivalent circuit can be used also for fitting dynamic impedance spectra.

The cyclic voltammogram performed during DMFA is shown in Figure 5, cleaned up from the multisine and compared to the steady-state voltammetry recovered from the set of stationary impedance spectra. The shape

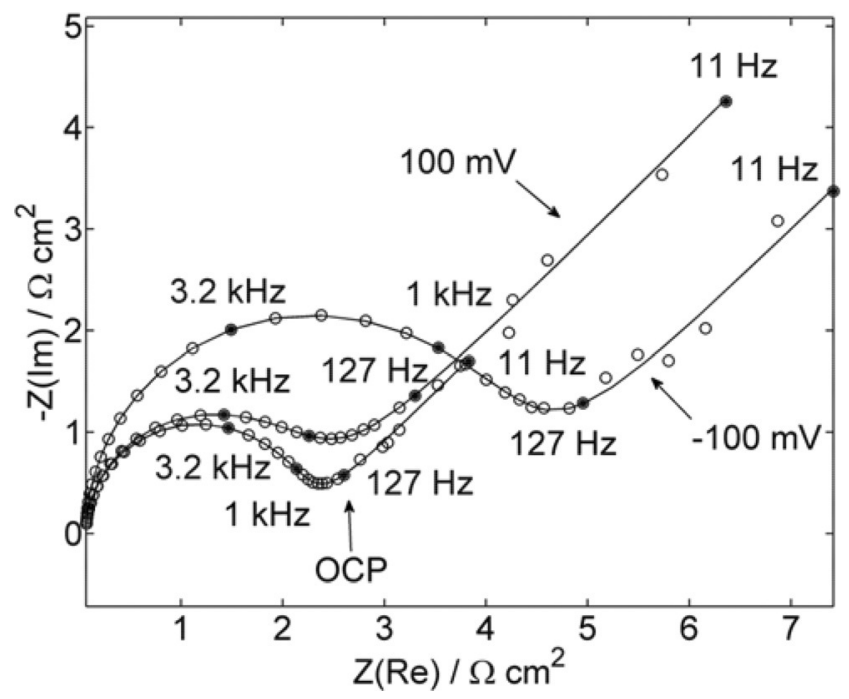

Fig. 4. Nyquist plot for three dynamic impedances with corresponding fitting $-100 \mathrm{mV}, 0$, and $100 \mathrm{mV}$. The impedances correspond to the cathodic scan.

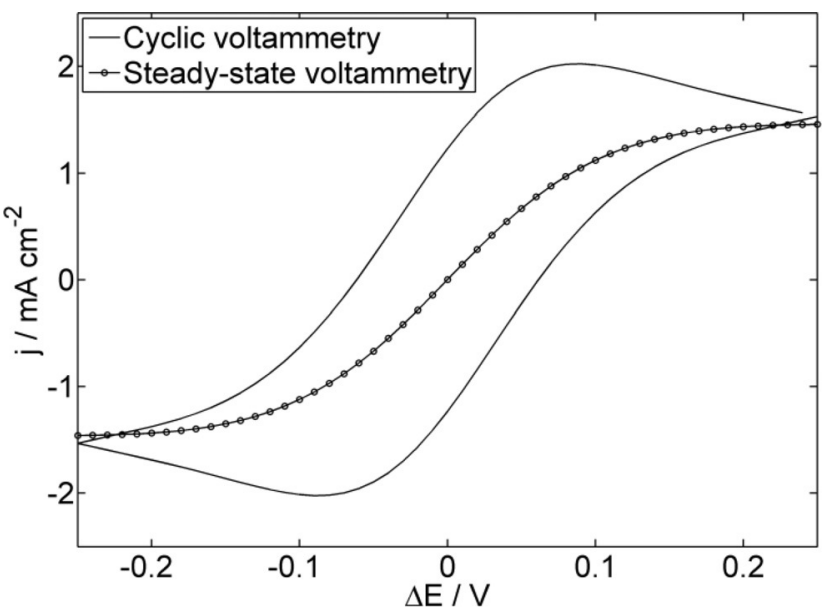

Fig. 5. Cyclic voltammetry cleaned up from the multisine signals. Scan rate $100 \mathrm{mV} \mathrm{s}^{-1}$.

of the $\mathrm{CV}$ is that of a mass transport limited process which approaches the limiting diffusion current at the extremes.

The value of the fitting parameters for stationary and dynamic (both anodic and cathodic scan) impedance spectra is presented in Figure 6. 41 spectra have been fitted simultaneously, between $-200 \mathrm{mV}$ and $+200 \mathrm{mV}$, one every $10 \mathrm{mV}$. In all cases $\mathrm{w}_{\mathrm{P}}$ for $\mathrm{R}_{\mathrm{s}}$ was set equal to infinite and gave a value equal to $6.64 \cdot 10^{-2} \Omega \mathrm{cm}^{2}$ for the static impedances, and $6.51 \cdot 10^{-2} \Omega \mathrm{cm}^{2}$ and $6.48 \cdot 10^{-2} \Omega \mathrm{cm}^{2}$, for the cathodic and anodic scan, respectively.

$\mathrm{C}_{\mathrm{h}}$ showed a variation of $1 \%$. This parameter was allowed to change between the spectra, but with a strong constrain $\left(\mathrm{w}_{\mathrm{P}}=1000\right) . \mathrm{C}_{\mathrm{h}}$ is larger in the case of the dynamic impedance and shows a lower variation. As expected, the voltage profile of the cyclic voltammetry has small influence on the value of the double layer capacitance both in stationary and dynamic conditions.

$\mathrm{R}_{\mathrm{ct}}$ and $\sigma$ showed a clear trend even with a low smoothing constrain $\left(\mathrm{w}_{\mathrm{P}}=0.1\right.$ or 0$)$ in all cases. This trend was already reported elsewhere $[10,25]$ and it is given by the changing of the concentration of the redox species at the interface. $\sigma$ has a minimum in correspondence of $E_{1 / 2}$ which is influenced by the ratio of the diffusion coefficients of the redox couple, while the minimum of $\mathrm{R}_{\mathrm{ct}}$ is determined also by the symmetry factor of the reaction. As expected, $\mathrm{R}_{\mathrm{ct}}$ and $\sigma$ do not overlap neither between stationary and dynamic impedance nor between cathodic and anodic scan.

In fact, in the dynamic impedance the system is not in steady-state conditions, therefore the concentration of redox species at the electrode and the diffusion profile depends on the direction of the scan and on the scan rate. The diffusion profile determines the value of $\mathrm{c}_{\mathrm{ox}}$ and $\mathrm{c}_{\mathrm{red}}$ in the reaction subdomains (II and IV), and as consequence it will influence the Warburg element and the charge transfer resistance. Note that the same phenomena 
a)

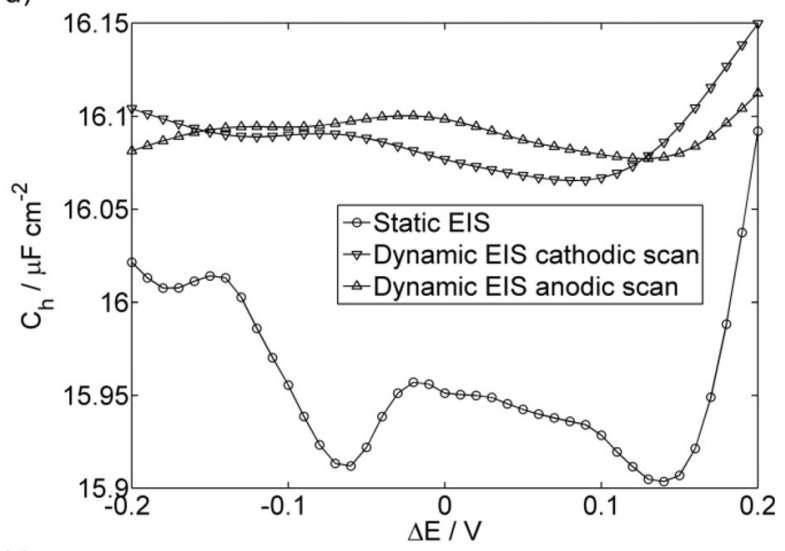

b)

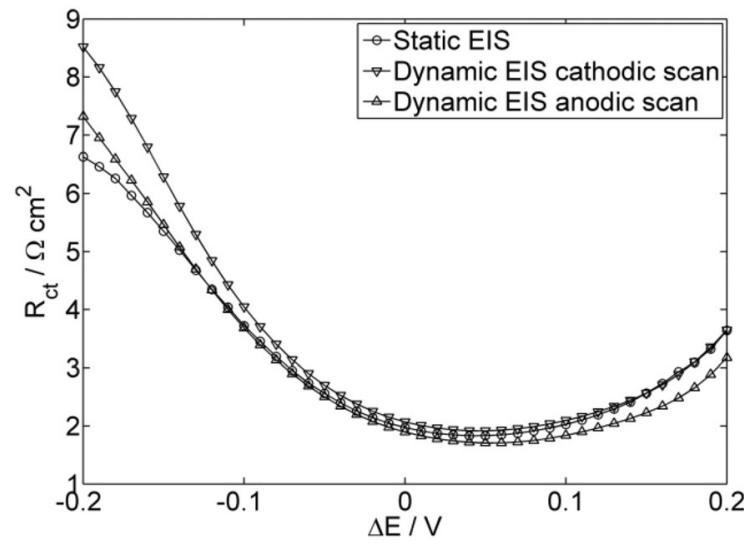

c)

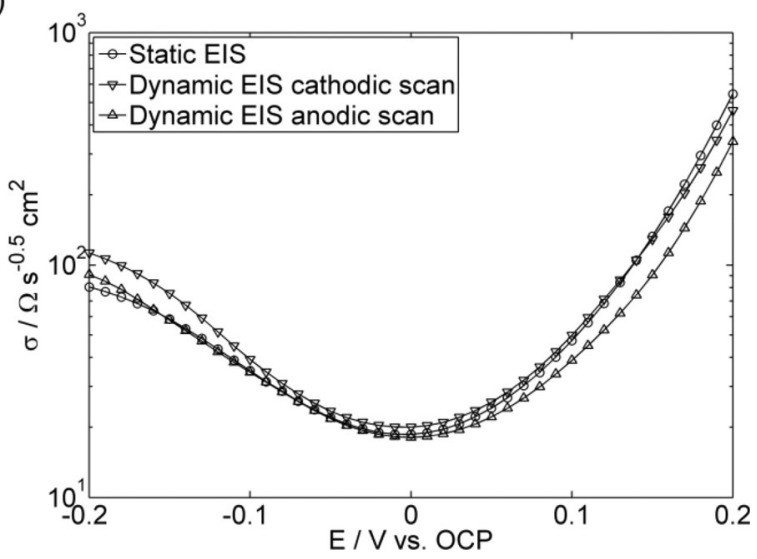

Fig. 6. Comparison of $\mathrm{C}_{\mathrm{h}}, \mathrm{R}_{\mathrm{ct}}$, and $\sigma$ derived from the fitting of the static impedance and for the dynamic impedance. a) Double layer capacitance $C_{h}$; b) charge transfer resistance $R_{c t}$; and $c$ ) Warburg element $\sigma$

give rise to the differences between the cyclic and the steady-state voltammogram of Figure 4

Anyway, the difference of the value of the fitting parameters extrapolated from the different experiments is small enough that the standard procedure of impedance analysis is not affected. It is important to underline the fact that the possibility to use the same equivalent circuit for stationary and dynamic impedance spectra is extremely valuable. In fact, one could use the same model and equivalent circuit for studying a reaction in dynamic con- ditions, observe how the parameters of the circuit change with time/potential, and develop a mechanism explaining the time evolution.

\section{Conclusions}

In this work multisine cyclic voltammetry was compared with standard stationary impedance spectroscopy on the ground of a simulated system consisting of a redox couple in solution in contact with a platinum electrode. In both stationary and dynamic case it was possible to study the system in terms of a Randles circuit, proving that conventional equivalent circuits can be employed also in nonstationary systems. However, there are some differences in the trends of the fitting parameters. In fact, although following the same behavior, the parameters of the circuit represent a different temporal situation.

The mathematical base for dynamic impedance is also reported in this work to conciliate the time dependence with the concept of impedance. The advantage of the dynamic multi-frequencies analysis (DMFA) applied to a cyclic voltammetry is that it is possible to collect the information derived from a standard cyclic voltammetry and implement them with those usually derived from impedance spectroscopy in a single experiment, i.e. it is possible to study the dynamic system in a large range of time constants simultaneously. The large amount of data can be analyzed with the algorithm proposed in this manuscript, in order to maintain the correlation between consecutive impedance spectra. This is obtained by introducing additional constrains on the smoothness of the time variation of the fitting parameters. This is a flexible way to keep the interdependence of all the parameters without the burden of assuming a particular trend.

The cyclic voltammetry was taken as starting point to show the possibility to recover meaningful impedance spectra in dynamic conditions. In this case, in fact, the dynamic behavior of the system is manifested through the variation of concentration of redox species at the interface, however the same principle can be applied in the case of evolution of the reaction, as in corrosion, electrocatalysis, or batteries, with the time. Other interesting application fields for the DMFA would be the study of systems showing hysteresis behavior [26,27], to observe, for example, which parameters control the hysteresis, and in the case of memory effects $[28,29]$. In both cases dynamic conditions are essential to picture properly the phenomenology of the system.

\section{Acknowledgements}

The financial support of the Federal Ministry of Education and Research (BMBF) in the framework of the project "Energiespeicher" (FKZ 03K3005) are gratefully acknowledged. 


\section{Full Paper}

\section{References}

[1] G. A. Ragoisha, A. S. Bondarenko, N. P. Osipovich, E. A. Streltsov, J. Electroanal. Chem. 2004, 565, 227-234.

[2] J. Házì, D. M. Elton, W. A. Czerwinski, J. Schiewe, V. A. Vicente-Beckett, A. M. Bond, Journal of Electroanalytical Chemistry 1997, 437, 1-15.

[3] A. R. Harris, J. Zhang, A. Konash, D. Elton, M. Hyland, A. M. Bond, J Solid State Electrochem 2008, 12, 1301-1315.

[4] A. Konash, A. R. Harris, J. Zhang, D. Elton, M. Hyland, G. Kennedy, A. M. Bond, J Solid State Electrochem 2008, 13, $551-563$.

[5] M. J. Walters, J. E. Garland, C. M. Pettit, D. S. Zimmerman, D. R. Marr, D. Roy, Journal of Electroanalytical Chemistry 2001, 499, 48-60.

[6] C. M. Pettit, J. E. Garland, N. R. Etukudo, K. A. Assiongbon, S. B. Emery, D. Roy, Applied Surface Science 2002 $202,33-46$.

[7] K. Darowicki, J. Orlikowski, G. Lentka, Journal of Electroanalytical Chemistry 2000, 486, 106-110.

[8] K. Darowicki, Journal of Electroanalytical Chemistry 2000, 486, 101-105.

[9] K. Darowicki, J. Orlikowski, A. Arutunow, Electrochimica Acta 2003, 48, 4189-4196.

[10] K. Darowicki, P. Ślepski, Journal of Electroanalytical Chemistry 2003, 547, 1-8.

[11] K. Darowicki, P. Ślepski, M. Szociński, Progress in Organic Coatings 2005, 52, 306-310.

[12] R. L. Sacci, D. Harrington, ECS Trans. 2009, 19, 31-42.

[13] R. L. Sacci, D. Harrington, ECS Trans. 2009, 19, 123-129.

[14] D. A. Harrington, ECS Trans. 2013, 45, 3-14.

[15] R. L. Sacci, F. Seland, D. A. Harrington, Electrochimica Acta 2014, 131, 13-19.
[16] E. Van Gheem, R. Pintelon, J. Vereecken, J. Schoukens, A. Hubin, P. Verboven, O. Blajiev, Electrochimica Acta 2004 49, 4753-4762.

[17] E. Van Gheem, R. Pintelon, A. Hubin, J. Schoukens, P. Verboven, O. Blajiev, J. Vereecken, Electrochimica Acta 2006 , $51,1443-1452$.

[18] Y. Van Ingelgem, E. Tourwé, O. Blajiev, R. Pintelon, A. Hubin, Electroanalysis 2009, 21, 730-739.

[19] T. Breugelmans, J. Lataire, T. Muselle, E. Tourwé, R. Pintelon, A. Hubin, Electrochimica Acta 2012, 76, 375-382.

[20] L. Fernández Macía, M. Petrova, A. Hubin, Journal of Electroanalytical Chemistry 2015, 737, 46-53.

[21] E. Madej, S. Klink, W. Schuhmann, E. Ventosa, F. La Mantia, Journal of Power Sources 2015, 297, 140-148.

[22] A. Battistel, F. La Mantia, Anal. Chem. 2013, 85, 67996805.

[23] A. Battistel, F. La Mantia, Electrochimica Acta 2015, 176, 1484-1491.

[24] A. Battistel, A. Petkovic, F. La Mantia, Electrochimica Acta 2015, 176, 1492-1499.

[25] B. E. Conway, J. O. Bockris, R. E. White, Modern Aspects of Electrochemistry, Plenum, New York; London, 1999.

[26] W. Dreyer, J. Jamnik, C. Guhlke, R. Huth, J. Moškon, M. Gaberšček, Nat Mater 2010, 9, 448-453.

[27] M. Gebala, F. La Mantia, W. Schuhmann, ChemPhysChem 2013, 14, 2208-2216.

[28] T. Sasaki, Y. Ukyo, P. Novák, Nat Mater 2013, 12, 569-575.

[29] E. Madej, F. La Mantia, W. Schuhmann, E. Ventosa, Adv. Energy Mater. 2014, 4, 1400829.

Received: April 16, 2016

Accepted: June 9, 2016 Published online: July 19, 2016 